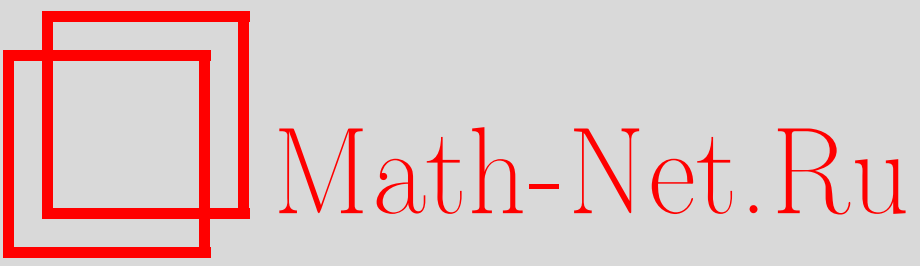

В. С. Ярунин, Низкотемпературная квазиклассика для квантовых макроскопических эффектов, ТМФ, 1999, том 119, номер 2, 308-331

DOI: https://doi.org/10.4213/tmf741

Использование Общероссийского математического портала Math-Net.Ru подразумевает, что вы прочитали и согласны с пользовательским соглашением

http://www.mathnet.ru/rus/agreement

Параметры загрузки:

IP: 52.6 .47 .48

26 апреля 2023 г., 17:00:10 
ТЕОРЕТИЧЕСКАЯ

И МАТЕМАТИЧЕСКАЯ

ФИЗИКА

Том 119, № 2

май, 1999

(C) 1999 г.

В. С. Ярунин*

\title{
НИЗКОТЕМПЕРАТУРНАЯ КВАЗИКЛАССИКА ДЛЯ КВАНТОВЫХ МАКРОСКОПИЧЕСКИХ ЭФФЕКТОВ
}

\begin{abstract}
Рассматриваются квантовые системы с двумя масштабами времени для "быстрых" (квантовых) и "медленных" (квазиклассических) степеней свободы с частотами колебаний $\Omega$ и $\omega \ll \Omega$, соответственно. Статистическая сумма представлятся в виде интеграла по траекториям с использованием как обычных когерентных состояний для бозонов и фермионов, так и их обобщения для случая нетривиальной алгебры наблюдаемых. Вычисление интеграла по "быстрым" траекториям приводит к нелокальному (по мнимому времени) эффективному действию для "медленных" (квазиклассических) переменных, которое принимает локальную форму в низкотемпературном приближении. Это низкотемпературное адиабатическое приближение выражается неравенством $\beta \Omega \gg 1 \gg \omega / \Omega$ и служит основой для квазиклассического описания в представлении интегралов по траекториям. В рамках этой концепции рассмотрены три сложные системы физики конденсированных сред так, что "медленные" и "быстрые" переменные представлены локализованными и зонными электронами в решетчатой модели Андерсона, бозонами конденсата и "надконденсата" в модели Боголюбова (с нарушенной трансляционной симметрией) и длинноволновыми фононами и электронами в больших молекулах при электрон-фононных оптических переходах. Соответствующие макроскопические квантовые явления представляют собой кондо-реорганизацию спектра коррелированных электронов, бозе-конденсацию в неоднородной среде и хаотизацию вибронного спектра молекул.
\end{abstract}

\section{1. ВВЕДЕНИЕ}

Метод интегралов по траекториям служит удобным средством для построения квазиклассического приближения в квантовой механике и статистической физике [1]. Он описывает высоковозбужденные квантово-механические, а также высокотемпературные квантово-статистические состояния. Помимо этого, можно построить низкотемпературное квазиклассическое приближение для сложных систем физики конденсированных сред, обнаруживаюших макроскопические квантовые эффекты при низких темпера-

* Объединенный институт ядерных исследований, Дубна, Россия.

E-mail: yarunin@thsun1.jinr.dubna.su 
турах. Целью данной работы является обоснование в рамках адиабатической картины в “температурном времени" подхода, основанного на разделении "медленных" и "быстрых" степеней свободы системы с частотами $\omega$ и $\Omega \gg \omega$, соответственно. В терминах квазиклассической нелинейной двухмасштабной по времени динамики такое разделение удобно сформулировать для интегралов по траекториям в когерентном [2] представлении. Усреднение по “быстрым" переменным с энергиями возбуждений (частотами) $\Omega$ приводит к движению "медленных" переменных, описываемому эффективным действием с запаздыванием по времени. Локальное по времени описание этого движения справедливо в случае больших $\Omega$, так что условие малых температур $\beta \Omega \gg 1$ обеспечивает "медленное” адиабатичаское квазиклассическое движение, свойственное квантовым макроскопическим явлениям. Непертурбативный анализ “медленной” подсистемы является составным элементом адиабатического приближения.

В работе предложенный подход применен к трем физическим примерам. Эти примеры, рассмотренные в разделах 3,4 и 5, касаются электронной проводимости, бозе-конденсации атомов и электрон-фононного взаимодействия в больших молекулах.

Общая схема низкотемпературного адиабатического приближения изложена в разделе 2. Она основана на применении непертурбативных функциональных методов вычисления интегралов по траекториям в представлении когерентных состояний $[3,4]$. Показано, что запаздываюшее (нелокальное по времени) действие для “медленных" переменных путем соответствуюшего квазиклассического анализа приближенно может быть приведено к локальной форме.

Эффект Кондо для магнитной восприимчивости, обусловленный рассеянием зонных электронов на локализованных, наблюдался в редкоземельных материалах [5]. Периодическая модель Андерсона для этих двух типов электронов использована в разделе 3 при описании этого эффекта. В пункте 3.1 для "медленных" локализованных электронов использовано $S U(2 \mid 1)$-когерентноепредставление хаббардовских переменных. Адиабатическое рассмотрение локализованных электронов как “медленных" переменных для статистической суммы, представленной интегралом по траеториям, обнаруживает в системе температурную аномалию кондо-типа (пункт 3.2 ).

Идея Боголюбова [6] о с-числовом описании бозе-эйнштейновской конденсации обсуждается в адиабатическом приближении в духе двухмасштабной по импульсам динамики бозонов [7]. В пункте 4.2 рассмотрение обобшено для систем с нарушенной трансляционной инвариантностью. Представлено низкотемпературное приближение для

"медленных" частищ конденсата и “быстрых" надконденсатных частиц. В пункте 4.3 это приближение применено к сверхтекучему жидкому ${ }^{4}$ Не в пористых стеклах при сравнении его с аналогичным эффектом (пункт 4.1) для сплошной среды. Кроме того, в пункте 4.3 рассматривается зависимость бозе-конденсации идеального газа от типа атомной ловушки и обсуждается низкотемпературный макроскопический квантовый эффект для плотности надконденсатных состояний в ней.

При электронных оптических переходах в больших молекулах значительная часть 
энергии возбуждений передается фононной подсистеме, движение длинноволновой части которой, как показано в разделе 5 , имеет квазиклассический характер. Электрон-фононное взаимодействие (пункт 5.1) и необратимая нелинейная динамика фононов (пункт 5.2) приводят к экспериментально наблюдаемому размытию оптического спектpa.

В заключении приводятся краткий обзор квазиклассических идей в методе интеграла по траекториям и сопутствуюшие комментарии.

\section{2. ЗАПАЗДЫВАЮШЕЕ ДЕЙСТВИЕ ДЛЯ “МЕДЛЕННЫХ" ПЕРЕМЕННЫХ}

Обратимся к сложным квантовым системам, содержащим "медленные" $(F)$ и "быстрые" (b) переменные. Основные свойства рассматриваемых систем могут быть описаны гамильтонианом

$$
H=\sum_{r} \omega_{r} F_{r}^{0}+\sum_{r} \Omega_{r} b_{r}^{+} b_{r}+\sum_{r} g_{r}\left[\left(b_{r}^{+}\right)^{m} F_{r}^{-}+F_{r}^{+}\left(b_{r}\right)^{m}\right], \omega \ll \Omega .
$$

Эта модель охватьвает три типа систем при значениях $m$ и $F$, выбранных следующим образом:

1) периодическая модель Андерсона для локализованных $(F)$ и зонных $(b)$ электронов; $r$ - индекс узла решетки, $m=1, b$ - фермионы, $F$ - хаббардовские переменные;

2 ) взаимодействие боголюбовского типа между классическими ( $F=$ const $)$ частицами и бозонами $(b) ; r$ - индекс квантового состояния, $m=2$;

3 ) электрон $(b)$-фононное $(F)$ взаимодействие в молекуле; $r$ - номер атома, $m=2$, $F-$ бозоны.

Эти системы будут рассмотрены в разделах 3,4 и 5, но предварительно основная идея будет пояснена в общей форме для (1).

Мы вычисляем статистическую сумму

$$
Q=\operatorname{Tr} \exp (-\beta H)=\int D \mu_{F} D \mu_{b} e^{S(0, \beta)}
$$

системы $S$ с действием

$$
S(0, \beta)=\int_{0}^{\beta} L d \tau, \quad L=L_{F}+L_{b}+L_{F, b}, \quad L_{b}=-b^{*} K_{\Omega} b, \quad K_{\Omega}=\frac{d}{d t}+\Omega,
$$

$r$-столбцовые обозначения опушены. Среднее по переменным $b, b^{*}$ может быть вычислено с периодическими (бозоны, $\kappa=1$ ) и антипериодическими (фермионы, $\kappa=-1$ ) граничными значениями, так что эффективное действие $S_{\mathrm{ef}}(F)$ определяется формулами

$$
\begin{aligned}
Q & =\int D \mu_{F} e^{S_{\mathrm{ef}}}, \quad e^{S_{\mathrm{ef}}}=\int D \mu_{b} e^{S(0, \beta)}, \\
S_{\mathrm{ef}} & =S_{F}-\kappa \ln \left(\operatorname{Det} K_{\Omega}\right)+\int_{0}^{\beta} F^{*} K_{\Omega}^{-1} F d \tau, \\
K_{\Omega}^{-1} & =\int_{0}^{\beta} d t^{\prime} e^{\Omega\left(t^{\prime}-t\right)}\left[\theta\left(t-t^{\prime}\right)+\left(\kappa e^{\Omega \beta}-1\right)^{-1}\right], \quad \operatorname{Tr} K_{\Omega}^{-1}=\beta\left(\kappa e^{\beta \Omega}-1\right)^{-1}
\end{aligned}
$$


для $m=1$. Отношение детерминантов вычисляется по формуле [3]

$$
\left(\frac{\operatorname{Det} K_{\Omega}}{\operatorname{Det} K_{\Omega_{0}}}\right)^{\kappa}=\exp \int_{\Omega_{0}}^{\Omega} \operatorname{Tr} K_{x}^{-1} d x=\frac{1-\kappa e^{-\beta \Omega}}{1-\kappa e^{-\beta \Omega_{0}}}
$$

Что касается случая $m=2$, то предметом рассмотрения будут парные коррелящии бозонов $b^{2} \rightarrow b_{k} b_{-k}$, так что

$$
S=\frac{1}{2} \int_{0}^{\beta}\left(b_{k}^{*} b_{-k}\right) M\left(\begin{array}{c}
b_{k} \\
b_{-k}^{*}
\end{array}\right) d t, \quad D \mu_{b}=D b_{k}^{*} D b_{k}
$$

и формула для отношения детерминантов имеет вид [4]

$$
\left(\frac{\operatorname{Det} M_{0}}{\operatorname{Det} M}\right)^{\kappa}=\exp \left[-\int_{0}^{g} \operatorname{Tr}\left(M^{-1} \frac{\partial M}{\partial x}\right) d x\right], \quad M=\left(\begin{array}{cc}
K_{\Omega} & -g F \\
-g F^{*} & -K_{-\Omega}
\end{array}\right) \text {. }
$$

Решение уравнения для траекторий приводит к оператору $M^{-1}$ :

$$
\begin{aligned}
\left(\begin{array}{c}
b_{k} \\
b_{-k}^{*}
\end{array}\right) & =M^{-1}\left(\begin{array}{c}
A \\
A^{*}
\end{array}\right)= \\
& =\exp \left(-\int_{0}^{t} m d \tau\right)\left[\int_{0}^{t} \exp \left(\int_{0}^{\tau} m d \tau^{\prime}\right)\left(\begin{array}{c}
-A \\
A^{*}
\end{array}\right) d \tau+\left(\begin{array}{c}
c \\
c^{*}
\end{array}\right)\right], \\
m & =\left(\begin{array}{cc}
\Omega & g F \\
-g F^{*} & -\Omega
\end{array}\right) .
\end{aligned}
$$

Оба уравнения (3) и (4) содержат нелокальные (по мнимому времени) запаздывающие члены в $S_{\text {ef }}$ с операторами $K^{-1}$ и $M^{-1}$, обусловленными дифференцированием $M \sim$ $K \sim d / d t$ траекторий $F$. Если предположить "медленность" траекторий $F$, то имеет место спектральное представление

$$
\left(K_{\Omega}^{-1}\right)_{n}=\frac{1}{-i \Omega_{n}+\Omega} \simeq \frac{1}{\Omega}, \quad \Omega_{n}=\frac{\pi}{\beta}\left\{\begin{array}{l}
\frac{2 n}{\beta}, \quad \text { если } \kappa=1, \\
\frac{2 n+1}{\beta}, \text { если } \kappa=-1
\end{array}\right.
$$

$(\kappa-$ индекс статистики переменной $F)$, обусловленное неравенствами

$$
\beta \Omega \gg 1 \gg \frac{\omega}{\Omega}
$$

требуюшими, чтобы величина $d F / d t \simeq \omega$ была пренебрежимо мала. Таким образом, выполняются приближения

$$
\int_{0}^{\beta} F^{*} K_{\Omega}^{-1} F d \tau \simeq \frac{|F|^{2} \beta}{\Omega}, \quad\left(\begin{array}{c}
c \\
c^{*}
\end{array}\right) \simeq\left(\kappa e^{\beta m}-1\right)^{-1} \int_{0}^{\beta} e^{m \tau}\left(\begin{array}{c}
-A \\
A^{*}
\end{array}\right) d \tau
$$


в формулах (3), (4), где для $b$ заданы периодические (антипериодические) граничные условия. После несложных преобразований мы получаем окончательный результат для $m=2$ в виде

$$
\begin{gathered}
\frac{\operatorname{Det} M_{0}}{\operatorname{Det} M}=\frac{\operatorname{sh}^{2}(\beta \Omega / 2)}{\operatorname{sh}^{2}(\beta E / 2)}, \quad E=\sqrt{\Omega^{2}-(g|F|)^{2}}, \quad \frac{1}{\operatorname{Det} M}=\frac{\exp (\Omega \beta)}{4 \operatorname{sh}^{2}(\beta E / 2)}, \quad \kappa=1, \\
\frac{\operatorname{Det} M_{0}}{\operatorname{Det} M}=\frac{1+\operatorname{ch}(\beta \Omega)}{\operatorname{ch}(\beta \Omega)+\operatorname{ch}(\beta g|F|)}, \quad \operatorname{Det} M=2 e^{-\beta \Omega}[\operatorname{ch}(\beta \Omega)+\operatorname{ch}(\beta g|F|)], \quad \kappa=-1, \\
S_{\text {ef }}=S_{a}-\kappa \ln (\operatorname{Det} M) .
\end{gathered}
$$

Условия (5) выражают адиабатическое приближение для переменных статистики Гиббса во "мнимом времени". Эти условия позволяют устранить запаздывание в эффективном действии $S_{\mathrm{ef}}$ и приводят к гамильтоновой теории с энергией $H_{\mathrm{ef}}(F)$,

$$
Q=\int D \mu_{F} e^{S_{\mathrm{ef}}} \simeq \operatorname{Tr} e^{-\beta H_{\mathrm{ef}}} .
$$

Правая часть неравенства (5) выражает адиабатическое приближение, левая часть квазиклассическое приближение, обусловленное как низкой температурой, так и малой массой $m$ в частоте $\Omega=h / 2 m$ “быстрых" частиц (раздел 3 ), ненулевым импульсом спектра Боголюбова $E_{k}$ (раздел 4 ) и малой частотой $\omega$ деформационных молекулярных фононов (раздел 5). Квазиклассическая сущность приближения (5) и последней формулы основана на большей близости “медленных" переменных к постоянным по сравнению с "быстрыми" траекториями, представляющими квантовые степени свободы. Заметим еще раз, что адиабатическое приближение (5) есть также низкотемпературное приближение, и это обстоятельство можно подчеркнуть, написав неравенство (5) в виде $\Omega^{2} \gg \omega T$.

\section{3. ЛОКАЛИЗОВАННЫЕ ЭЛЕКТРОНЫ В ПЕРИОДИЧЕСКОЙ МОДЕЛИ АНДЕРСОНА}

Двухмасштабная во времени динамика в периодической модели Андерсона образуется двумя типами электронов: локализованными электронами (описываемыми хаббардовскими $X$-операторами) и зонными электронами (описываемыми фермионными операторами $\left.b, b^{+}\right)$в гамильтониане

$$
H=\sum_{r \sigma}(E-\mu) X_{r}^{\sigma \sigma}-\mu \sum_{i \sigma} n_{i \sigma}^{b}+g \sum_{r \sigma}\left(b_{r \sigma}^{+} X_{r}^{0 \sigma}+X_{r}^{\sigma 0} b_{r \sigma}\right)-t \sum_{\langle r s\rangle \sigma} b_{r \sigma}^{+} b_{s \sigma} .
$$

Здесь $t$ - константа перескока локализованных электронов, $g$ - взаимодействие между ними и зонными электронами, $E<0$ - уровень энергии локализованных электронов. Операторы Хаббарда определены следуюшим образом:

$$
X^{\sigma 0}=|\sigma\rangle\left\langle 0\left|, \quad X^{\sigma \sigma^{\prime}}=\right| \sigma\right\rangle\left\langle\sigma^{\prime}\right|,
$$

где $|0\rangle$ означает свободный узел (дырку) и $|\sigma\rangle$ есть состояние, занятое электроном со спином $\sigma$. Условия $\sigma= \pm, \sigma \neq 2$ в гамильтониане (8) подразумевают одночастичное заполнение узлов решетки, что соответствует большим $(U \gg t)$ хаббардовским энергиям $U$, опушенным в формуле (8). 
3.1. $S U(2 \mid 1)$-представление для хаббардовских переменных. Алгебра $X$-операторов однозначно идентифицируется с представлением $s u(2 \mid 1)$-супералгебры,

$$
\begin{gathered}
Q_{3}=\frac{1}{2}\left(X^{++}-X^{--}\right), \quad Q_{+}=X^{+-}, \quad Q_{-}=X^{-+}, \quad B=\frac{1}{2}\left(X^{++}+X^{--}\right)+X^{00}, \\
V_{+}=X^{0-}, \quad V_{-}=-X^{0+}, \quad W_{+}=X^{+0}, \quad W_{-}=X^{-0}
\end{gathered}
$$

с четными $\left(B, Q_{3}, Q_{+}, Q_{-}\right)$и нечетными $\left(W_{+}, W_{-}, V_{+}, V_{-}\right)$генераторами [8, 9]. Следуя работам $[10,11]$, мы используем суперсимметричное $S U(2 \mid 1)$-представление хаббардовских переменных в интеграле по траекториям для статистической суммы $Q$ и обычные грассмановы образующие $b_{r \sigma}^{*}, b_{r \sigma}$ для зонных электронов:

$$
\begin{aligned}
Q= & \operatorname{Tr} \exp (-\beta H)= \\
= & \int \prod_{r \sigma} d \mu_{r} d b_{r \sigma}^{*} d b_{r \sigma} \exp \left[\frac{1}{2} \sum_{r} \int_{0}^{\beta} \frac{\dot{z}_{r}^{*} z_{r}-z_{r}^{*} \dot{z}_{r}+\dot{\xi}_{r}^{*} \xi_{r}-\xi_{r}^{*} \dot{\xi}_{r}}{1+\left|z_{r}\right|^{2}+\xi_{r}^{*} \xi_{r}} d t+\right. \\
& +\frac{1}{2} \sum_{r \sigma} \int_{0}^{\beta}\left(\dot{b}_{r \sigma}^{*} b_{r \sigma}-b_{r \sigma}^{*} \dot{b}_{r \sigma}\right) d t-\sum_{r} \int_{0}^{\beta} h\left(z_{r}^{*}, \xi_{r}^{*}, z_{i}, \xi_{r}\right) d t+ \\
& \left.+\int_{0}^{\beta}\left(t \sum_{\langle r s\rangle} b_{r \sigma}^{*} b_{s \sigma}+\mu \sum_{r \sigma} b_{r \sigma}^{*} b_{r \sigma}\right) d t\right], \\
h\left(z_{r}^{*}, \xi_{r}^{*}, z_{r}, \xi_{r}\right)= & \frac{\left\langle z_{r}, \xi_{r}|h| z_{r}, \xi_{r}\right\rangle}{\left\langle z_{r}, \xi_{r} \mid z_{r}, \xi_{r}\right\rangle}= \\
= & \frac{(E-\mu)\left(1+\left|z_{r}\right|^{2}\right)}{1+\left|z_{r}\right|^{2}+\xi_{r}^{*} \xi_{r}}+g \frac{b_{i \uparrow}^{*} \xi_{r}^{*}+z_{r \downarrow}^{*} \xi_{r} z_{r}+\xi_{r} b_{i \uparrow}+z_{r}^{*} \xi_{r} b_{i \downarrow}}{1+\left|z_{r}\right|^{2}+\xi_{r}^{*} \xi_{r}} .
\end{aligned}
$$

Суперкогерентные состояния $|z, \xi\rangle$ нумеруются координатами на сфере обобщенного фазового пространства $z, \xi$, соответствуюшего генераторам $Q_{-}$и $V_{-} . S U(2 \mid 1)$-инвариантная мера интегрирования в (9) имеет вид

$$
d \mu_{r}=\frac{d z_{r}^{*} d z_{r}}{2 \pi i} \frac{d \xi_{r}^{*} d \xi_{r}}{1+\left|z_{r}\right|^{2}+\xi_{i}^{*} \xi_{r}}
$$

С помошью двух последовательных преобразований в (9) можно одновременно привести меру (10) и кинетический член $S U(2 \mid 1)$ к виду, соответствующему независимым спину $S U(2)$ и бесспиновому фермиону

$$
\begin{gathered}
z \rightarrow z \sqrt{1+\xi^{*} \xi}, \quad \xi \rightarrow \xi \sqrt{1+|z|^{2}} \\
d \mu_{r} \rightarrow \frac{d z_{r}^{*} d z_{r} d \xi_{r}^{*} d \xi_{r}}{2 \pi i\left(1+\left|z_{r}\right|^{2}\right)^{2}}, \quad S_{\mathrm{kin}} \rightarrow \frac{1}{2} \sum_{r} \frac{\dot{z}_{r}^{*} z_{r}-z_{r}^{*} \dot{z}_{r}}{1+\left|z_{r}\right|^{2}}+\frac{1}{2} \sum_{r}\left(\dot{\xi}_{r}^{*} \xi_{r}-\xi_{r}^{*} \dot{\xi}_{r}\right) .
\end{gathered}
$$

Как видно из (11), грассмановы траектории $\xi_{r}^{*}, \quad \xi_{r}$ соответствуют бесспиновым переменным и удовлетворяют антипериодическим граничным условиям $\xi(0)=-\xi(\beta)$, 
$\xi^{*}(0)=-\xi^{*}(\beta)$, в то время как комплексные траектории $z_{r}^{*}, z_{r}$ с периодическими граничными условиями $z(0)=z(\beta), z^{*}(0)=z^{*}(\beta)$ соответствуют спиновым переменным $X$-подсистемы. Таким образом, амплитуда $d_{\sigma} d$-электрона разложена по полям $\xi$ и $z$, представляюшим зарядовую и спиновую степени свободы, соответственно.

Интеграл по траекториям $b$ гауссов, он вычисляется в явном виде по формуле (3). Функциональный детерминант второй вариации действия $b$-электрона не зависит от $X$-переменных. В результате мы получаем следуюшее эффективное действие для подсистемы локализованных $X$-электронов:

$$
\begin{aligned}
S_{\mathrm{ef}}^{X}= & \frac{1}{2} \sum_{r} \int_{0}^{\beta} \frac{\dot{z}_{r}^{*} z_{r}-z_{r}^{*} \dot{z}_{r}}{1+|z|^{2}} d t+\frac{1}{2} \sum_{r} \int_{0}^{\beta}\left(\dot{\xi}_{r}^{*} \xi_{r}-\xi_{r}^{*} \dot{\xi}_{r}\right) d t+ \\
& +N(E-\mu)-(E-\mu) \sum_{r} \int_{0}^{\beta} \xi_{r}^{*} \xi_{r} d t+ \\
& +g^{2} \sum_{\langle r s\rangle} \int_{0}^{\beta} \int_{0}^{\beta} d t d t^{\prime} \xi_{r}(t) \xi_{s}^{*}\left(t^{\prime}\right) G_{r s}\left(t-t^{\prime}\right)\left(z_{r}(t) \mid z_{s}\left(t^{\prime}\right)\right) .
\end{aligned}
$$

Заметим, что последний член в эффективном действии (12) записан в виде $S U(2)$-матричного элемента

$$
\left(z_{r}(t) \mid z_{s}\left(t^{\prime}\right)\right)=\frac{1+z_{r}^{*}(t) z_{s}\left(t^{\prime}\right)}{\sqrt{\left(1+\left|z_{r}(t)\right|^{2}\right)\left(1+\left|z_{s}\left(t^{\prime}\right)\right|^{2}\right)}}
$$

Функция Грина $G_{r s}\left(\tau-\tau^{\prime}\right)$ есть пропагатор $b$-электронов,

$$
\begin{aligned}
G_{r s}\left(\tau-\tau^{\prime}\right) & =\frac{1}{N \beta} \sum_{q, n} \frac{\exp \left[-i \omega_{n}\left(\tau-\tau^{\prime}\right)+i q(r-s)\right]}{i \omega_{n}+t_{q}-\mu}, \\
\omega_{n} & =\frac{2 n+1}{\beta} \pi, \quad t_{q}=-t \sum_{Z} e^{i q \triangle_{Z}},
\end{aligned}
$$

где $\triangle_{Z}$ - расстояние между узлами решетки, $Z$ - число ближайших соседей для узла. Следует отметить, что эффективное действие (12) нелокально по времени, как и $S_{\mathrm{ef}}$ в формуле (3), так что никакое гамильтоново движение не может быть ассоциировано с действием (12).

3.2. Адиабатическое приближение для температурной аномалии Кондо. Обратимся к адиабатическому приближению (5) для системы (8). Из формулы (14) следует, что эффективное действие (12) принимает локальный вид

$$
Q \simeq \operatorname{Tr} \exp \left(-\beta H_{\mathrm{ef}}^{X}\right),
$$

если выполняется неравенство

$$
|\beta(\Omega-\mu)| \gg 1,
$$


где $\Omega=Z t$ - полуширина зоны проводимости. В этом случае в низкотемпературном приближении получаем

$$
G \rightarrow \delta\left(\tau-\tau^{\prime}\right) \frac{1}{N} \sum_{q} \frac{\exp [i q(r-s)]}{t_{q}-\mu}
$$

и последнее слагаемое в формуле (12) для эффективного действия $S_{\mathrm{ef}}^{X}$ принимает вид

$$
\frac{g^{2}}{N} \sum_{\langle r s\rangle} \int_{0}^{\beta} d t \xi_{r}(t) \xi_{s}^{*}(t) \sum_{q} \frac{\exp [i q(r-s)]}{t_{q}-\mu}\left(z_{r}(t) \mid z_{s}(t)\right) .
$$

Статический вариант среднего поля для спиновых переменных в выражении (16) приводит к приближению $\left(z_{r}(t) \mid z_{s}(t)\right) \rightarrow 1$. В результате получается эффективный гамильтониан для $X$-электронов

$$
H_{\mathrm{ef}}^{X}=\left(E_{\mathrm{ef}}-\mu\right) \sum_{r} d_{r}^{+} d_{r}-\sum_{\langle r s\rangle} t_{r s}^{\mathrm{ef}} d_{r}^{+} d_{s}
$$

где $d_{i}^{+}, d_{i}$ - операторы рождения и уничтожения бесспиновых фермионов и

$$
E_{\mathrm{ef}}=E-\frac{g^{2}}{N} \sum_{q} \frac{1}{t_{q}-\mu}, \quad t_{i j}^{\mathrm{ef}}=\frac{1}{N} \sum_{q} \frac{\exp [i q(r-s)]}{t_{q}-\mu} .
$$

Неравенство (15) представляет собой левую часть неравенства (5), в то время как правая часть (5) в данном случае принимает вид

$$
|E-\mu| \ll \Omega=Z t
$$

Последнее неравенство есть условие "медленности" $X$-электронов. Это означает, что $X$-уровень является мелким по сравнению с полушириной $\Omega$ зоны $b$-электронов. Неравенство (18) регулирует знак сдвига энергии $\delta E X$-электронов в зоне проводимости $b$-электронов. В низкотемпературном пределе сдвиг энергии $\delta E$ в зоне проводимости $b$-электронов двумерного проводника с плотностью состояний $\rho_{b}(\epsilon)=(1 / 2 \Omega) \theta\left(\Omega^{2}-\epsilon^{2}\right)$ имеет вид

$$
\delta E=-g^{2} \int \frac{\rho_{b}}{\epsilon-\mu} d \epsilon, \quad \rho_{b}=\frac{1}{2 \Omega} .
$$

В рамках низкотемпературного адиабатического приближения и приближения среднего поля (17) для $X$-электрона ситуации с глубоким $(\Omega \ll|E|)$ или мелким $(\Omega \gg|E|)$ $d$-электронными уровнями приводят к различным случаям. Они соответствуют узкой или широкой зонам $b$-электронов с различными интервалами $\epsilon$ в интеграле (19). Таким образом, формула

$$
\delta E=-\frac{g^{2}}{2 \Omega}\left\{\int_{-\Omega}^{\mu-T}+\int_{\mu+T}^{\Omega}\right\} \frac{d \epsilon}{\epsilon-\mu}=-\frac{g^{2}}{2 \Omega} \ln \left|\frac{\Omega-\mu}{\Omega+\mu}\right|<0
$$


имеет место при условиях $E>\mu, \Omega \ll|E|, T<|E|$ и электронном числе заполнения $N<1$. Далее, адиабатическое приближение (15) для мелкого $X$-уровня приводит к формуле

$$
\delta E=-\frac{g^{2}}{2 \Omega} \int_{-\Omega}^{\Omega-2 T} \frac{d \epsilon}{\epsilon-\mu} \simeq-\frac{g^{2}}{2 \Omega} \ln \left|\frac{2 T}{\Omega+\mu}\right|>0
$$

при условиях $|E| \ll \Omega, T<\Omega, N>1$. Перестройка спектра происходит при "температуре Кондо"

$$
T_{k}=\frac{\Omega+\mu}{2} \exp \left(\frac{E-\mu}{g^{2} \rho_{b}}\right),
$$

определяемой соотношением $E-\mu+\delta E=0$.

Таким образом, мы показали, что суперкогерентное $S U(2 \mid 1)$-представление хаббардовских операторов в интеграле по траекториям для статистической суммы периодической модели Андерсона (8) в пределе $U \rightarrow \infty$ приводит к разделению зарядовой и спиновой переменных для $X$-электронов. Эти переменные представлены траекториями $z$ и $\xi$, соответствуюшими $S U(2)$-спину и бесспиновому фермиону. Приближения низких температур и спинового среднего поля для такого представления удовлетворяют адиабатической аппроксимации (см. (15), (18)))

$$
\beta|\Omega-\mu| \gg 1 \gg \frac{|E-\mu|}{\Omega}
$$

для $X$-электронов в статистической сумме системы (8) и приводят к эффективному гамильтониану (17). С двиг $\delta E$ уровня $X$-электрона вычисляется усреднением по степеням свободы $b$-электронов. При этом возможны две разные ситуации для случаев глубокого $(\Omega \ll|E|, \quad N<1)$ и мелкого $(\Omega \gg|E|, \quad N>1)$ уровней $X$-электронов. Независимость величины $\delta E(20)$ от температуры обнаружена в первом случае. Во втором случае $\delta E$ имеет кондовский тип поведения по температуре $\delta E \sim \ln T$.

Известно [5], что кондовская температурная аномалия свидетельствует об изменении проводимости, связанном со свойством $X$-электронов становиться “тяжелыми" частицами, что сопровождается пиком $X$-электронной плотности $\rho_{x}$. Это было подтверждено нахождением [11] для системы (17) пика $\rho_{x}(y), \quad y=\beta|\mu-\Omega|$ в точке $y=1.7$. "Тяжелые" электроны "медленной” $X$-подсистемы проявляют макроскопический эффект в модели (8) при условии (23), являюшемся условием низкотемпературной квазиклассики в духе неравенств (5).

\section{4. БОЗЕ-ЭЙНШТЕЙНОВСКАЯ КОНДЕНСАЦИЯ}

Известная модель Боголюбова [6] была первой микроскопической теорией бозе-эйнштейновской конденсации неидеального газа. В ней использовались $c$-числовое описание конденсата $(k=0)$ и операторы стандартного пространства Фока для надконденсатных бозонов. Действие для системы бозонов со взаимодействием $G\left(r-r^{\prime}\right)$ между 
ними в общем случае имеет вид

$$
\begin{aligned}
S & =\int_{0}^{\beta} d t\left[\int d r \psi(r, t) \frac{\partial}{\partial t} \psi^{*}(r, t)-H(t)\right], \quad r=(x, y, z), \quad H=H_{0}+h, \\
H_{0} & =\int d r\left[\frac{1}{2 m}\left(\nabla \psi^{*}(r, t) \nabla \psi(r, t)\right)+\psi^{*} U(r) \psi\right], \\
h & =\frac{1}{2} \int d r d r^{\prime} G\left(r-r^{\prime}\right) \psi^{*}(r, t) \psi^{*}\left(r^{\prime}, t\right) \psi\left(r^{\prime}, t\right) \psi(r, t),
\end{aligned}
$$

где $U$ - потенциал внешнего поля. Заметим, что $c$-сдвиг [12] амплитуды бозе-операторов

$$
\psi^{+}(r, t)=B_{0}^{*}+B^{+}(r, t), \quad\left[\psi(r), \psi^{+}\left(r^{\prime}\right)\right]=\delta\left(r-r^{\prime}\right)
$$

приводит к модели Боголюбова [6] с квадратичной операторной формой относительно операторов $B, B^{+}$и с квадратичной $c$-числовой формой относительно переменных $B_{0}, B_{0}^{*}$.

4.1. Низкотемпературное приближение и квазиклассика. Заметим, что две компоненты $B_{0}, B$ бозе-поля $\psi(r, t)$ можно рассматривать как переменные двух подсистем в смысле адиабатического приближения раздела 2. При этом правая часть неравенства (5) обеспечивается условием $\omega \sim d / d t \sim 0$ для "медленной" подсистемы конденсата, поскольку амплитуды $B_{0}, B_{0}^{*}$ постоянны. Левая часть (5) обусловлена низкотемпературным неравенством $\beta E_{k} \gg 1$, где $E_{k}$ - боголюбовский спектр надконденсатных возбуждений, так что неравенство (5) принимает вид

$$
\beta E_{k} \gg 1 \gg \frac{\omega}{E_{k}} \simeq 0
$$

Очевидно, что можно точно вычислить след в статистической сумме модели Боголюбова по надконденсатным переменным. В методе интеграла по траекториям это усреднение приводит к эффективному действию $S_{\text {ef }}(\rho)$ для плотности конденсата $\rho=\left|B_{0}\right|^{2}$. Ниже кратко будут представлены основные этапы этого рассмотрения.

Вариационные уравнения $\delta S_{\mathrm{ef}}=0$ как приближенные для квазиклассического параметра $\rho(T)$ в пределе $N, V \rightarrow \infty$ для сверхтекучего ${ }^{4}$ Не дают

$$
\begin{aligned}
R & =\rho+r, \quad \frac{\mu}{g_{0}}-2 r=\rho\left[1-\frac{D(\rho, \mu)}{g_{0}}\right] \\
D & =\frac{c}{2} \int k^{2} \operatorname{cth}\left(\frac{\beta E_{k}}{4}\right) \frac{g_{k}^{2}}{E_{k}} d k>0, \quad r=\frac{c}{2} \int k^{2} \Phi d k \\
\Phi & =\frac{\omega_{k}-\nu}{E_{k}} \operatorname{cth}\left(\frac{\beta E_{k}}{4}\right)-1
\end{aligned}
$$

где $R$ - полная плотность ${ }^{4} \mathrm{He}, r$ - надконденсатная плотность, $\mu$ - химический потенциал бозонов. 
Эти уравнения были выведены и исследованы в работах $[13-15]$ для жидкого ${ }^{4}$ Не в отсутствие $(U=0)$ внешнего поля при подходяшем выборе межбозонного взаимодействия. Между теоретическим $E_{k}$ и экспериментальньм спектрами $[16,17]$ обнаруживается согласие.

Теплоемкость

$$
C_{v}=-T \frac{\partial^{2} F}{\partial T^{2}}, \quad F=-\frac{1}{\beta} S_{\mathrm{ef}}
$$

может быть вычислена аналитически [18] с использованием приближенных решений $[15,19]$ вариационного уравнения $\delta S_{\text {ef }}=0$. Различия между низкотемпературными $\left(T<T_{\mathrm{c}}\right)$ и высокотемпературными $\left(T>T_{\mathrm{c}}\right)$ решениями вариационных уравнений обнаруживаются благодаря наличию $[13-15,18]$ "квазиклассического" интеграла движения

$$
\frac{d}{d t}\left[B_{0}^{*} B_{0}+\int B^{+}(r) B(r) d r\right]=0
$$

для системы $H=H_{0}+h$. Согласие между теоретическими [18] и экспериментальными [20] значениями $C_{v}$ удовлетворительное при $0<T<1.5 K$ и $T>2.3 K$, но не в окрестности критической температуры $T_{\mathrm{c}}$.

Масштаб энергии надконденсатных возбуждений $E_{k}$ в ${ }^{4}$ Не виден из значения максонного максимума спектра $\left.E_{k}\right|_{\max } \simeq 15 K$ нейтронного рассеяния [16]. Это означает, что неравенству для параметра $y=\beta E_{k}>4$ удовлетворяют все значения переменных, кроме высоких температур и малых импульсов $E_{k}<4 T$. Для безразмерного параметpa $y$

$$
\begin{aligned}
& 4<y<y_{0}, \quad T>T_{\mathrm{c}}, \\
& y_{0}<y<\infty, \quad T<T_{\mathrm{c}}
\end{aligned}
$$

граничное значение $y_{0}$ найдено в работе [18] эмпирически при сравнении экспериментальных и теоретических значений $C_{v}$ и равно 4.4 .

Ввиду большого интереса к бозе-конденсации ${ }^{4}$ Не в пористых средах и парах щелочных металлов в магнитных ловушках мы в следуюшем пункте обратимся к приближению Боголюбова в системах с нарушенной трансляционной симметрией, которая присуща обоим упомянутым случаям.

4.2. Модель Боголюбова бозе-конденсации в трансляционно-неинвариантных системах. Проделаем $c$-сдвиг (24) амплитуд бозе-операторов

$$
\begin{aligned}
\psi^{+}(r, t) & =\frac{1}{\sqrt{V}} \sum_{n=0} b_{n}^{+}(t) u_{n}(r)=\frac{1}{\sqrt{V}}\left(\sum_{n \neq 0} b_{n}^{+}(t) u_{n}(r)+b_{0}^{*} u_{0}(r)\right) \\
b_{0} & =\sqrt{N_{0}} e^{i \alpha}, \quad n=\left(n_{x}, n_{y}, n_{z}\right), \quad \int d r u_{n} u_{n^{\prime}}=\delta_{n, n^{\prime}}
\end{aligned}
$$


с использованием трехмерных одночастичных собственных функций $u_{n}$, являющихся решениями уравнений для бозона

$$
\left\{\nabla^{2}+\frac{2 m}{h^{2}}\left[E_{n}-U(r)\right]\right\} u_{n}=0
$$

с уровнями энергии $E_{n}$. В случае фурье-разложения $u_{n} \rightarrow \exp (i k r)$ вклад в боголюбовскую энергию можно классифицировать следующим образом:

а) для обычных членов $\left(k_{1}+k_{2}=k_{3}+k_{4}\right)-$

$$
\begin{gathered}
G\left(r-r^{\prime}\right) B_{0}^{*} B_{0} B_{0}^{*} B_{0} \\
G\left(r-r^{\prime}\right)\left[B^{*}(r) B(r) B_{0}^{*} B_{0}+B^{*}\left(r^{\prime}\right) B\left(r^{\prime}\right) B_{0}^{*} B_{0}+\text { h.c. }\right]
\end{gathered}
$$

б) для слагаемых с нарушенной градиентной инвариантностью $\left(k_{1}+k_{2}=k_{3}+k_{4}\right)-$

$$
G\left(r-r^{\prime}\right)\left[B^{*}(r) B^{*}\left(r^{\prime}\right) B_{0} B_{0}+B(r) B\left(r^{\prime}\right) B_{0}^{*} B_{0}^{*}\right]
$$

в) в случае нарушения градиентной и транслящионной симметрий -

$$
\begin{aligned}
G\left(r, r^{\prime}\right) & {\left[B^{*}(r) B_{0}^{*} B_{0} B_{0}+B\left(r^{\prime}\right)^{*} B_{0}^{*} B_{0} B_{0}+\text { h.c. }\right] \rightarrow } \\
& \rightarrow\left\{\begin{array}{l}
\langle k, 0|G| 0,0\rangle, \quad k_{1}+k_{2} \neq k_{3}+k_{4}, \quad G=G\left(r, r^{\prime}\right), \\
0, \quad k_{1}+k_{2}=k_{3}+k_{4}, \quad G=G\left(r-r^{\prime}\right),
\end{array}\right.
\end{aligned}
$$

после вычисления интегралов по $r, r^{\prime}$.

В обшем случае использование собственных функций $u_{n}$ приводит к приближению боголюбовского вида со взаимодействием $h_{B}$ между бозонами

$$
\begin{aligned}
& S_{B}=\int_{0}^{\beta}\left(L_{0}-H_{0}-h_{B}\right) d t, \quad L_{0}=\int d r B \frac{\partial B^{*}}{\partial t}, \quad h_{B}=\frac{1}{2} \int d r d r^{\prime} \epsilon(t) \\
& H_{0}=\int d r\left[\frac{1}{2 m}\left(\nabla \psi^{*} \nabla \psi\right)+U(r)\left(\frac{N_{0}}{V} u_{0}^{2}+\sqrt{\frac{N_{0}}{V}}\left(B e^{-i \alpha}+B^{*} e^{i \alpha}\right) u_{0}+B^{*} B\right)\right]= \\
& =\frac{N_{0} w_{00}}{V}+\frac{1}{V} \sum_{n, n^{\prime} \neq 0} b_{n}^{*} b_{n^{\prime}} w_{n n^{\prime}}+\sqrt{\frac{N_{0}}{V}} \sum_{n \neq 0}\left(b_{n} e^{i \alpha}+b_{n}^{*} e^{-i \alpha}\right) w_{0 n} \\
& \epsilon(t)= \\
& \quad G\left(r-r^{\prime}\right)\left\{\frac{N_{0}^{2}}{V^{2}} u_{0}^{2}(r) u_{0}^{2}\left(r^{\prime}\right)+\frac{N_{0}^{3 / 2}}{V^{2}} \sum_{n \neq 0} u_{0}(r) u_{0}\left(r^{\prime}\right)^{2} u_{n}(r)\left(b_{n} e^{-i \alpha}+b_{n}^{*} e^{i \alpha}\right)+\right. \\
& +\frac{2 N_{0}}{V^{2}} \sum_{n, n^{\prime} \neq 0}\left[u_{n}\left(r^{\prime}\right) u_{n^{\prime}}\left(r^{\prime}\right) u_{0}^{2}(r)+\right. \\
& +u_{n}\left(r^{\prime}\right) u_{n^{\prime}}(r) u_{0}^{2}(r)\left(b_{n}^{*} b_{n^{\prime}}^{*} e^{2 i \alpha}+u_{n} d r+\int(r) u_{n} u_{n^{\prime}} d r\right.
\end{aligned}
$$


Нарушение трансляционной инвариантности учитывается в линейных по $b$ операторных слагаемых в $H_{0}$ и $\epsilon(t), N_{0}$ есть число бозонов конденсата среди $N$ частиц системы. Одночастичная энергия $w_{n n^{\prime}}$ содержит как член кинетической энергии, пропорциональньй $\nabla^{2}$, так и энергию во внешнем поле, пропорциональную $U(r)$.

В разреженных газах применимо $\delta$-приближение для взаимодействия:

$$
\begin{gathered}
G\left(r-r^{\prime}\right) \rightarrow G \delta\left(r-r^{\prime}\right), \quad h_{B}=\frac{1}{2} \int d r d r^{\prime} \epsilon(t) \rightarrow h_{B}^{\delta}, \\
h_{B}^{\delta}=\frac{G}{2}\left[\frac{N_{0}^{2}}{V^{2}} \int d r u_{0}^{4}(r)+\frac{N_{0}^{3 / 2}}{V^{2}} \sum_{n \neq 0}\left(b_{n} e^{-i \alpha}+b_{n}^{*} e^{i \alpha}\right) \int d r u_{0}^{3}(r) u_{n}(r)+\right. \\
+\frac{2 N_{0}}{V^{2}} \sum_{n, n^{\prime} \neq 0} b_{n}^{*} b_{n^{\prime}} \int d r u_{0}^{2}(r) u_{n}(r) u_{n^{\prime}}(r)+ \\
\left.+\frac{N_{0}}{V} \sum_{n, n^{\prime} \neq 0} u_{0}^{2} u_{n}(r) u_{n^{\prime}}(r)\left(b_{n}^{*} b_{n^{\prime}}^{*} e^{2 i \alpha}+b_{n} b_{n^{\prime}} e^{-2 i \alpha}\right)\right] .
\end{gathered}
$$

В пределе $N, V \rightarrow \infty$ с масштабным преобразованием $b_{n} \rightarrow \sqrt{V} b_{n}, b_{n}^{*} \rightarrow \sqrt{V} b_{n}^{*}$ получаем

$$
\begin{aligned}
H_{0}= & \rho w_{00}+\sqrt{\rho} \sum_{n \neq 0}\left(b_{n} e^{-i \alpha}+b_{n}^{*} e^{i \alpha}\right) w_{0 n}+\sum_{n, n^{\prime} \neq 0} b_{n}^{*} b_{n^{\prime}} w_{n, n^{\prime}}, \\
h_{B}^{\delta}= & \rho^{2} \gamma_{0}+\rho^{3 / 2} \sum_{n \neq 0}\left(b_{n} e^{-i \alpha}+b_{n}^{*} e^{i \alpha}\right) \gamma_{0 n}+ \\
& +2 \rho \sum_{n, n^{\prime} \neq 0}\left[b_{n}^{*} b_{n^{\prime}}+\frac{1}{2}\left(b_{n}^{*} b_{n^{\prime}}^{*} e^{2 i \alpha}+b_{n} b_{n^{\prime}} e^{-2 i \alpha}\right)\right] \gamma_{n n^{\prime}},
\end{aligned}
$$

где

$$
\gamma_{0}=\frac{G}{2} \int d r u_{0}^{4}, \quad \gamma_{0 n}=\frac{G}{2} \int d r u_{0}^{3} u_{n}, \quad \gamma_{n n^{\prime}}=\frac{G}{2} \int d r u_{0}^{2} u_{n} u_{n^{\prime}},
$$

$\rho=N_{0} / V$ - плотность бозе-конденсата. Оператор $H_{0}$ - гамильтониан идеального бозегаза, оператор $h_{B}^{\delta}$ обобщает боголюбовское взаимодействие через парные корреляции в $\delta$-приближении, слагаемые с коэффициентами $\gamma_{0 n}$ и $w_{0 n}$ отвечают за нарушение трансляционной инвариантности.

4.3. Бозе-конденсация в пористых стеклах и в разреженных газах в ловушках. Общий анализ, предложенный в пунктах 4.1 и 4.2, был применен в работе [21] для пространственно-неоднородной сверхтекучей системы [22]. Сравнивались вариационные уравнения для трансляционно-инвариантной $G\left(r-r^{\prime}\right)$ и трансляционно-неинвариантной $G\left(r, r^{\prime}\right)$ систем с целью оценить влияние нарушения трансляционной инвариантности на температуру $T_{\text {c }}$ сверхтекучего перехода. Матричный элемент $G_{k, 0}=$ $\langle k, 0|G| 0,0\rangle$ в соотношениях (28), описывающий взаимодействие между конденсатными и надконденсатными частицами, ответствен за сдвиг $T_{\mathrm{c}}$. Параметр $x$ :

$$
x=\frac{Y}{G_{0}}, \quad Y=\frac{c}{2} \int k^{2} \frac{\left|g_{k, 0}\right|^{2}}{E_{k}} d k, \quad c^{-1}=2 \pi^{2} h^{3}, \quad G_{0}=\langle 0,0|G| 0,0\rangle,
$$


есть мера этого влияния. С помошью формул (30), (31) экспериментальное [22] отношение температур перехода в однородном $\left(\left.T_{\mathrm{c}}\right|_{x=0}\right)$ случае и в пористой среде $\left(T_{\mathrm{c}}\right)$ было представлено [21] как отношение плотностей бозе-конденсата

$$
\frac{T_{\mathrm{c}}}{\left.T_{\mathrm{c}}\right|_{x=0}} \simeq \frac{\rho}{\left.\rho\right|_{x=0}}=1-\alpha(x)<1
$$

Вычисление $\alpha(x)$ в формуле (32) приводит к оценке $x \simeq 0.1$, которая показывает $10 \%$-ный вклад стенок пор в искажение потенциальной энергии $G\left(r-r^{\prime}\right) \rightarrow G\left(r, r^{\prime}\right)$.

Что касается бозе-конденсации атомов металлов в ловушках, то если пренебречь потенциалом взаимодействия $G$, квазиклассические свойства заметны благодаря присутствию потеншиала ловушки $U(r)$. Бозе-конденсат идеального газа в ловушке с любым потенциалом описывается гамильтонианом (30).

Можно привести поучительное вычисление числа надконденсатных атомов для такого случая [23], когда

$$
\begin{aligned}
& H_{0}-\rho w_{00}=\sum_{n, n^{\prime} \neq 0}\left(w_{n n} b_{n}^{*} b_{n}+b_{n} J_{n n^{\prime}}^{*}+b_{n}^{*} J_{n n^{\prime}}\right), \\
& J_{n n^{\prime}}=j_{n}+b_{n^{\prime}}\left(1-\delta_{n n^{\prime}}\right) w_{n n^{\prime}}, \quad j_{n}=\sqrt{\rho} w_{0 n} e^{i \alpha} .
\end{aligned}
$$

Статистическая сумма системы имеет вид

$$
\begin{aligned}
& Q_{0} e^{-\rho w_{00}}=\operatorname{Sp} e^{-\beta H_{0}}=\prod_{n, n^{\prime} \neq 0} \int d \rho \int D^{2} b_{n} D^{2} b_{n^{\prime}} \exp S_{n n^{\prime}}(0, \beta)= \\
&=\int d \rho \prod_{n, n^{\prime} \neq 0} \int D^{2} b_{n^{\prime}} \frac{\exp \left(\int_{0}^{\beta} J_{n n^{\prime}}^{*}\left(\frac{d}{d \tau}+w_{n n}\right)^{-1} J_{n n^{\prime}} d t\right)}{1-\exp \left(-\beta w_{n n}\right)}, \\
&\left(\frac{d}{d \tau}+w_{n n}\right)^{-1}=\int_{0}^{\beta} e^{w_{n n}(\tau-t)}\left[\theta(t-\tau)+\left(e^{\beta w}-1\right)^{-1}\right] d \tau .
\end{aligned}
$$

Воспользуемся квазиклассическим рассмотрением, принимая во внимание, что $d / d t \ll$ $w_{n n}$ приближенно выполняется при квазиклассическом движении системы. В этом случае формула (33) дает

$$
\mathrm{Sp} e^{-\beta H_{0}} \simeq \int d \rho e^{S_{\mathrm{ef}}}=\int d \rho \prod_{n, n^{\prime} \neq 0} \frac{1}{1-e^{-\beta w_{n n}}} \int D^{2} b_{n^{\prime}} \exp \left(\frac{\beta}{w_{n n}} J_{n n^{\prime}}^{*} J_{n n^{\prime}}\right) .
$$

Число надконденсатных частищ представляется выражением

$$
\begin{aligned}
\left.\left\langle b_{n}^{+} b_{n}\right\rangle\right|_{n \neq 0} & \sim \frac{\partial \ln \left(\exp S_{\mathrm{ef}}\right)}{\partial\left(-\beta w_{n n}\right)} \simeq \\
& \simeq \frac{1}{\exp \left(\beta w_{n n}\right)-1}+\frac{\left|j_{n}\right|^{2}}{w_{n n}^{2}}+\sum_{n \neq n^{\prime}} f\left(\beta w_{n n^{\prime}}, \frac{w_{n n^{\prime}}}{w_{n n}}\right), \\
f(a, b) & =\frac{b^{2}}{\exp (a, b)-1} .
\end{aligned}
$$

6 Теоретическая и математическая физика, т. 119, № 2, 1999 г. 
Последним слагаемым в формуле (34) можно пренебречь при условии $w_{n n^{\prime}} \ll w_{n n}$. Первое слагаемое в (34) дает главный вклад в величину $\left.\left\langle b_{n}^{+} b_{n}\right\rangle\right|_{n \neq 0}$ при температу$\operatorname{pax}$, близких к критической $T_{\mathrm{c}}$, поскольку $\left|j_{n}\right| \sim w_{0 n} \sqrt{\rho}$ и $\rho(\beta)$ обрашается в нуль при $T=T_{\text {c. }}$ Для параболической ловушки

$$
U_{\omega}(r)=\frac{m \omega^{2}}{2} r^{2}
$$

этот "высокотемпературный” квазиклассический член приводит к значению $\left.\left\langle b_{n}^{+} b_{n}\right\rangle\right|_{n \neq 0} \sim(T)^{3}$, что соответствует результату, полученному в работе [24]. Напротив, второе слагаемое в формуле (34) происходит от множителя $w_{0 n}$ и дает большой вклад при малых температурах, являясь результатом с-сдвига (24) в условиях нарушенной трансляционной симметрии. Общие неравенства (5) для этой низкотемпературной квазиклассики имеют вид

$$
\beta w_{n n} \gg 1 \simeq \frac{w_{0 n}}{w_{n n^{\prime}}} \gg \frac{w_{0 n}}{w_{n n}}
$$

Если мы обратимся к теплоемкости идеального газа в ловушке, то заметим, что она имеет различную температурную зависимость для различных значений $w_{n n^{\prime}}$ в различных внешних полях. Поэтому можно различать типы ловушек по измерениям $C_{v}$ для бозе-газа в них.

Таким образом, “квазиклассическая" плотность конденсата $\rho$ в приближении Боголюбова приводит к температурнонезависимому вкладу $\left|j_{n}\right|^{2} / w_{n n}^{2}$ в число надконденсатных частиц. Этот вклад, обусловленньй нарушением трансляционной симметрии, может быть обнаружен при низких температурах как квантовое макроскопическоепроявление бозе-конденсации идеального газа в ловушке.

\section{5. КВАЗИКЛАССИКА ФОНОНОВ В БОЛЬШИХ МОЛЕКУЛАХ}

Двухатомная молекула представляет собой пример адиабатической системы с "быстрыми" (электроны) и “медленными" (ядра) степенями свободы. Движение этих подсистем имеет квантовый (кроме точек поворота колебаний ядер) характер. Иная ситуация возможна в многоатомных молекулах, где имеются два вида движения ядер - валентные $\Omega_{a, b}$ и деформационные $\omega \simeq \Omega_{a}-\Omega_{b} \ll \Omega_{a, b}$ колебания, при этом “мягким," $\omega$-колебательным, модам может быть присуш квазиклассический характер движения. В частности, электронный отклик при поглошении когерентного света большой молекулой приводит к возбуждению оптически активных "мягких" фононов $\omega$ вследствие электрон-ффононного взаимодействия. Люминесценция сопровождается перераспределением энергии между $\omega$ (деформационными) и $\Omega_{a, b}$ (валентными) степенями свободы благодаря фонон-фононному взаимодействию. Эти квазиклассические нелинейные фононные процессы в молекуле, соответствуюшие стадиям поглошения и люминесценции, выражают статистическую необратимость, описываемую с помощью интегрирования по замкнутому временному контуру. 
Неравенства $n>1$ и $n<1$ для числа заполнения "мягкой” фононной моды

$$
n=\left(e^{\omega \beta}-1\right)^{-1}
$$

определяют высоко- и низкотемпературные квазиклассические аппроксимации $\omega \beta<1$ и $\omega \beta>1$, соответственно. Адекватная нелинейная квазиклассическая динамика ядер может быть развита для любых значений $n$, однако, как будет показано, лишь низкотемпературному движению $n<1$ соответствуют финитные траектории в квазиклассическом фононном фазовом пространстве. Адиабатическое квазиклассическое условие (5) в этом случае выглядит как

$$
\Omega_{a, b} \beta>\omega \beta>1>\frac{\omega}{\Omega_{a, b}}
$$

и экспериментально проявляется в размытии $[25,26]$ квантовой структуры спектральных линий поглошения и люминесценции оптической спектроскопии. Теоретический анализ, представленный ниже, следует работам [27, 28].

5.1. Электрон-фононное взаимодействие. Возьмем двухатомную молекулу с двумя электронными уровнями $E_{1}<E_{2}$ и частотой колебаний ядер $\omega$. Электронный переход происходит вследствие дипольного взаимодействия $\lambda$ с заданным оптическим полем $\psi$. Гамильтониан электрон-фононной системы во внешнем поле имеет вид

$$
h_{0}=\left(\begin{array}{cc}
E_{2}+\omega\left(c^{+}+\Delta\right)(c+\Delta) & \lambda \psi \\
\lambda \psi^{*} & E_{1}+\omega c^{+} c
\end{array}\right), \quad\left[c, c^{+}\right]=1,
$$

где $\Delta$ - сдвиг потенциальной кривой верхнего электронного состояния. Матричный элемент электрон-фононного перехода в представлении интегралов по траекториям в случае точного резонанса $\arg \psi=i t\left(E_{2}-E_{1}+\Delta^{2} \omega\right)$ сводится к виду $[29,30]$

$$
T \exp \left[i \int_{0}^{t}\left(\begin{array}{cc}
\omega \Delta\left(c^{*}+c\right) & \lambda|\psi| \\
\lambda|\psi| & 0
\end{array}\right) d t\right]
$$

где произведение $\omega \Delta$ описывает электрон-фононное взаимодействие, в то время как $c(0)$ и $c^{*}(t)$ соответствуют начальному и конечному когерентным состояниям осциллятора.

В случае многоатомной молекулы $\omega$-колебание выделяется как оптически активное и рассматривается его взаимодействие с $N$ парами фононов, имеющих частоты $\Omega_{a, b}$. Основная идея состоит в том, что электронный оптический переход в молекуле вызывает быстрые возбуждения системы ядер. Затем эти возбуждения трансформируются в нерегулярное движение ядер вследствие непертурбативного нелинейного взаимодействия между фононами (с межмодовым колебательным ангармонизмом $\mu$ ). В итоге гамильтониан молекулы конструируется с помошью $(2 \times 2)$-матрицы электронной системы $(37)$ 
и $2 N+1$ бозе-операторов $a, b, c$ колебаний ядер в основном $(h)$ и возбужденном $\left(h_{\Delta}\right)$ электронных состояниях:

$$
\begin{aligned}
H & =\left(\begin{array}{ll}
1 & 0 \\
0 & 0
\end{array}\right) h_{\Delta}+\left(\begin{array}{ll}
0 & 0 \\
0 & 1
\end{array}\right)(h-E), \quad E=E_{2}-E_{1}, \\
h & =\omega c^{+} c+\sum_{g=1}^{N}\left(a^{+} b^{+}\right)_{g}\left(\begin{array}{cc}
\Omega_{a} & \mu c \\
\mu c^{+} & \Omega_{b}
\end{array}\right)\left(\begin{array}{l}
a \\
b
\end{array}\right)_{g}, \quad \Omega_{a, b} \gg \omega .
\end{aligned}
$$

Гамильтониан $h$ описывает резонансное взаимодействие $\Omega_{a}-\Omega_{b} \simeq \omega$ оптически активного колебания $\omega$ с $N$ парами франк-кондоновских колебаний $\Omega_{a, b}, \mu$ - постоянная взаимодействия между фононами. Сдвиг $\Delta$ координаты оптически активного колебания в верхнем электронном состоянии определен как

$$
h_{\Delta}=\left.h\right|_{c \rightarrow c+\Delta, c^{+} \rightarrow c^{+}+\Delta}
$$

При взаимодействии со слабым оптическим полем электрон-фононные возбуждения описываются корреляционной функцией

$$
\begin{aligned}
& K(t)=(\operatorname{Tr} \rho)^{-1} I(t), \quad I=\operatorname{Tr}\left[\rho e^{-i H t}\left(\begin{array}{ll}
0 & 0 \\
1 & 0
\end{array}\right) e^{i H t}\left(\begin{array}{ll}
0 & 1 \\
0 & 0
\end{array}\right)\right], \\
& K(t)=e^{-i E t} \operatorname{Tr}\left[e^{-h(\beta+i t)} e^{i h_{\Delta} t}\right], \quad \rho=e^{-\beta H}
\end{aligned}
$$

В формуле (38) выполнено усреднение по электронным переменным, при этом неунитарный характер эволюции ядер вытекает из-за различия гамильтонианов $h$ и $h_{\Delta}$.

5.2. Квазиклассическая хаотизация фононов. Теория необратимых процессов дает возможность классифицировать динамику фононов на различных стадиях оптического перехода. Она основана на предположении, что эволюция квантовой системы "вперед" и "назад" по оси времени описывается разными гамильтонианами, так что движение системы по замкнутому контуру времени неунитарно. Такая ситуация имеет место в процессе молекулярного поглощения и люминесценции. Этот механизм необратимого движения распространяет метод интегрирования по замкнутому контуру времени $[31,32]$ на вычисление статистической суммы в виде интеграла по траекториям. Нас интересует соответствие между необратимостью оптического электронного перехода и хаотизацией ядерных степеней свободы в больших молекулах $[25,26]$.

Для того чтобы избежать теории возмушений по сдвигу $\Delta$ и по константе ангармонического взаимодействия $\mu$ между модами, обратимся к функционально-интегральному представлению для функции $K(t)$

$$
\begin{aligned}
I(t) & =\int d^{2} z d^{2} z^{\prime} d^{2} z^{\prime \prime}\left\langle z\left|e^{i t\left(h_{\Delta}-E\right)}\right| z^{\prime}\right\rangle\left\langle z^{\prime}\left|e^{-\beta h}\right| z^{\prime \prime}\right\rangle\left\langle z^{\prime \prime}\left|e^{-i h t}\right| z\right\rangle= \\
& =\int d^{2} z d^{2} z^{\prime} d^{2} z^{\prime \prime} \int D^{2} \phi_{-} D^{2} \phi D^{2} \phi_{+} \exp (\Phi-i E t),
\end{aligned}
$$


где

$$
\begin{aligned}
\Phi & =i S^{+}(t, 0)+S(\beta, 0)-i S_{\Delta}^{-}(t, 0), \\
d^{2} z & =d^{2} z_{\mathrm{c}} \prod_{g=1}^{N}\left(d^{2} z_{a} d^{2} z_{b}\right)_{g}, \quad d^{2} z_{\kappa}=e^{-|\kappa|^{2}} \frac{d \kappa^{*} d \kappa}{i \pi}, \quad \kappa=a, b, c, \\
D^{2} \phi_{ \pm} & =D^{2} c_{ \pm} \prod_{g=1}^{N}\left(D^{2} a_{ \pm} D^{2} b_{ \pm}\right)_{g}, \quad D^{2} \phi=D^{2} c \prod_{g=1}^{N}\left(D^{2} a D^{2} b\right)_{g} .
\end{aligned}
$$

Действия $S^{+}$и $S_{\Delta}^{-}$соответствуют движению колебательной подсистемы молекулы от момента времени 0 до момента $t$ с гамильтонианом $h$, а далее от момента $t$ до момента 0 с гамильтонианом $h_{\Delta}$. Действие $S$ соответствует тепловому равновесному состоянию с температурой $\beta^{-1}$. Граничные условия для траекторий выбраны слудующим образом:

$$
\begin{aligned}
& a_{-}^{*}(t)=z_{a}^{*}, \quad a^{*}(\beta)=z_{a}^{\prime *}, \quad a_{+}^{*}(t)=z_{a}^{\prime \prime *}, \\
& a_{-}(0)=z_{a}^{\prime}, \quad a(0)=z_{a}^{\prime \prime}, \quad a_{+}(0)=z_{a},
\end{aligned}
$$

и аналогично для $b, b_{ \pm}, c, c_{ \pm}$.

Интеграл по переменным $c, c_{ \pm}, z_{\mathrm{c}}, z_{\mathrm{c}}^{\prime}, z_{\mathrm{c}}^{\prime \prime}$ в выражении для $I(t)$ вычисляется точно:

$$
\begin{gathered}
I(t)=n \exp (\omega \beta-i E t) \int d^{2} z_{a, b} d^{2} z_{a, b}^{\prime} d^{2} z_{a, b}^{\prime \prime} \int D^{2} \phi_{a, b}^{-} D^{2} \phi_{a, b} D^{2} \phi_{a, b}^{+} \exp S_{\mathrm{ef}}, \\
S_{\mathrm{ef}}=-i \int_{0}^{t} L_{-} d \tau+\int_{0}^{\beta} L d \tau+i \int_{0}^{t} L_{+} d \tau+F \\
L=\sum_{g=1}^{N}\left[a^{*}\left(-\frac{d}{d \tau}-w_{a}\right) a+b^{*}\left(-\frac{d}{d \tau}-w_{b}\right) b\right]_{g} \\
L_{ \pm}=\sum_{g=1}^{N}\left[a_{ \pm}^{*}\left(i \frac{d}{d \tau}-w_{a}\right) a_{ \pm}+b_{ \pm}^{*}\left(i \frac{d}{d \tau}-w_{b}\right) b_{ \pm}\right]_{g} \\
\int_{0}^{\beta} d \tau d \tau^{\prime} f(\tau) f\left(\tau^{\prime}\right)\left[n+\theta\left(\tau-\tau^{\prime}\right)\right]+i n e^{i w t} \int_{0}^{\beta} f d \tau \int_{t}^{0}\left(f_{-}^{*}-f_{+}^{*}\right) d \tau^{\prime}- \\
-i n e^{-i w t} \int_{0}^{\beta} f^{*} d \tau \int_{t}^{0}\left(f_{+}-f_{-}\right) d \tau^{\prime}+\int_{t}^{0} d \tau d \tau^{\prime}\left[n\left(f_{-}^{*}-f_{+}^{*}\right)_{\tau}\left(f_{+}-f_{-}\right)_{\tau^{\prime}}+\right. \\
\left.+f_{-}\left(\tau^{\prime}\right)\left(f_{+}^{*}-f_{-}^{*}\right) \theta\left(\tau-\tau^{\prime}\right)+f_{+}^{*}(\tau)\left(f_{-}-f_{+}\right)_{\tau^{\prime}} \theta\left(\tau^{\prime}-\tau\right)\right]- \\
-\mu \Delta \int_{0}^{\beta}\left(Q+Q^{*}\right) d \tau-i \mu \Delta \int_{0}^{t}\left(Q+Q_{-}^{*}-2 w \Delta\right) d \tau .
\end{gathered}
$$

Слагаемые в фазе $F$, билинейные по $f, f_{ \pm}$, совпадают с аналогичными членами у Швингера [32], однако вместо феноменологических источников $K_{ \pm}$этой работы здесь возникает замкнутая система нелинейных уравнений для траекторий

$$
\begin{array}{ccc}
f_{ \pm}=e^{i w r} Q_{ \pm}, & f=e^{w r} Q, & f^{*}=e^{-w r} Q^{*}, \\
Q_{+}=\sum_{g=1}^{N} q_{g}^{+}, & Q_{-}=w \Delta+\sum_{g=1}^{N} q_{g}^{-}, & Q=\sum_{g=1}^{N} q_{g},
\end{array}
$$




$$
q^{ \pm}=\mu a_{ \pm} b_{ \pm}^{*}, \quad q=\mu a b^{*} .
$$

Слагаемые, пропорциональные $\Delta$ и $\Delta^{2}$, описывают влияние электронного перехода на амплитуды ядер.

Воспользуемся теперь квазиклассическим приближением. Первый его шаг состоит в переходе к термодинамическому пределу $N \rightarrow \infty$ и вариационному принципу

$$
\delta S^{+}=0, \quad \delta S=0, \quad \delta S_{\Delta}^{-}=0 .
$$

Можно выписать и проанализировать соответствующую систему замкнутых вариационных уравнений для переменных $a_{ \pm}, b_{ \pm}, a, b$ и $a_{ \pm}^{*}, b_{ \pm}^{*}, a^{*}, b^{*}$. Можно заметить, что пары переменных $a_{ \pm}$и $a_{ \pm}^{*}, b_{ \pm}$и $b_{ \pm}^{*} ; a, b$ и $a^{*}, b^{*}$ не являются канонически-сопряженными, потому что обсуждаемая система уравнений и система уравнений аналогичного вида

$$
\delta\left(S^{+}\right)^{*}=0, \quad \delta S^{*}=0, \quad \delta\left(S_{\Delta}^{-}\right)^{*}=0
$$

не совпадают. Данное обстоятельство является результатом неунитарности эволюции, которая исчезает при $\Delta \rightarrow 0$. Следующий шаг квазиклассического приближения состоит в предположении, что при электронном переходе рождается много оптически активных фононов. Это условие может быть выражено неравенством $\Delta^{2} \gg 1$ (в атомных единицах) и удовлетворяется при малой частоте $w$, что соответствует деформационным степеням свободы движения ядер. Именно такая ситуация имеет место для молекул в экспериментах $[25,26]$, где наблюдались значения $\omega \sim 200 \mathrm{~cm}^{-1}$ и $\Omega_{a, b} \sim 1000 \mathrm{~cm}^{-1}$.

При анализе вариационных уравнений для траекторий следует сделать еще одно уточнение. Будут исследованы два альтернативных случая, соответствующих неравенствам

$$
\Delta^{2}>1>n=\left(e^{w \beta}-1\right)^{-1}, \quad \Delta^{2}>n>1 .
$$

Данные неравенства позволяют пренебречь взаимодействием фононов на температурной части эволюции. Это означает, что температура не должна заметно отличаться от обычной $T_{0}=300 \mathrm{~K}$. Замкнутое уравнение

$$
\frac{d^{2} u_{ \pm}}{d \tau^{2}}-x_{1,2} \mu^{2} u_{ \pm}\left(2\left|u_{ \pm}\right|^{2}+\varepsilon_{1,2}^{ \pm}\right)=0
$$

где

$$
\begin{gathered}
\varepsilon_{1}^{+}=L_{+}, \quad \varepsilon_{1}^{-}=L_{+}-\Delta^{2}, \quad \varepsilon_{2}^{ \pm}=L_{ \pm}-n^{2}|\tilde{\Delta}|^{2}, \\
x_{1,2}=\left\{\begin{array}{c}
-1, \text { если } n<1, \\
1, \text { если } n>1,
\end{array}\right. \\
L_{ \pm}=\mp\left(n_{b}(0)-n_{a}(0)\right)+\left(\frac{\Omega}{2 \mu}\right)^{2}, \quad \Omega=\omega-\Omega_{a}+\Omega_{b}, \\
u_{ \pm}=x_{1,2} v_{ \pm}^{*}, \quad u_{-}(0)=-u_{+}(0) e^{-w \beta}+i \Delta, \quad u_{-}(t)=-u_{+}(t)+i \Delta,
\end{gathered}
$$


получается для обобшенных амплитуд ядер

$$
\begin{aligned}
u_{+} & =-\int_{0}^{\tau} f_{+} d \tau^{\prime}+(n+1) \int_{0}^{t}\left(f_{+}-f_{-}\right) d \tau^{\prime}+\tilde{\Delta}(n+1), \\
u_{-} & =-\int_{0}^{\tau} f_{-} d \tau^{\prime}-n \int_{0}^{t}\left(f_{+}-f_{-}\right) d \tau-n \tilde{\Delta}+i \Delta \\
\tilde{\Delta} & =i \Delta\left(e^{i w t}-1\right) .
\end{aligned}
$$

Уравнение (40) описьвает квазиклассическое - усредненное по времени - движение фононов $\Omega_{a, b}$, обусловленное нелинейным фононным взаимодействием, рассмотренным после усреднения по электронным переменным (38). Такое же уравнение может быть получено для амплитуд $v_{ \pm}$. Определение функции $x_{1,2}$ является весьма важным, поскольку оно определяет каноническое сопряжение. Можно заметить, что определение величин $x_{1,2}$ заменяет определения $\left(a_{ \pm}^{*}\right)^{*}$ и $\left(b_{ \pm}^{*}\right)^{*}$. В частном случае больших температур $(n>1)$ можно заметить, что выполняется обычное условие канонического сопряжения $\left(a_{ \pm}^{*}\right)^{*}=a_{ \pm},\left(b_{ \pm}^{*}\right)^{*}=b_{ \pm}$. Это можно пояснить хорошо известным в спектроскопии фактом, что различие между поглощением и люминесценцией исчезает при достаточно высокой температуре, как будто сдвиг $\Delta$ становится малым и имеет место почти унитарная эволюция по замкнутому контуру времени.

Таким образом, знак “+” в уравнении (40) означает поглощение и знак “-” означает люминесценцию. Для случая низких температур $\left(x=-1, T<T_{0}\right)$ имеет место апериодическое решение $u_{ \pm}=\left|\varepsilon_{1}^{ \pm}\right|^{1 / 2} \mathrm{ch}^{-1}\left(\tau \mu\left|\varepsilon_{1}^{ \pm}\right|^{1 / 2}\right)$, для которого энергия нелинейного движения близка к нулю и $\varepsilon_{1}^{ \pm}<0$. Это решение описывает "солитоноподобные" возбуждения фононной системы с условием резонанса $\omega \leftrightarrow \Omega_{a}-\Omega_{b}$, выполняющимся при $\Delta \mu>\Omega$. Для случая больших температур $\left(x=1, T>T_{0}\right)$ имеет место апериодическое решение $\left|\varepsilon_{2}^{ \pm}\right|^{1 / 2} \operatorname{th}\left(\tau \mu\left|\varepsilon_{2}^{ \pm}\right|^{1 / 2}\right)$, для которого энергия нелинейного движения близка к нулю и $\varepsilon_{2}^{ \pm}<0$. Это решение описывает “кинкоподобные" возбуждения фононной системы с условием резонанса $w \rightarrow\left(\Omega_{a}-\Omega_{b}\right), \omega \leftarrow\left(\Omega_{a}-\Omega_{b}\right)$, выполняюшимся при $\Delta \mu n>\Omega$. Это решение не финитно на фазовой плоскости и распадная часть его траектории соответствует диссоциации.

Апериодические решения имеют бесконечный период, и в их окрестности происходит нерегулярное движение нелинейных осцилляторов. Это движение может объяснить отсутствие обычной фононной структуры электронных молекулярных спектров, наблюдавшееся в работах $[25,26]$. Такая ситуация имеет место при $\Delta \mu>\Omega$ или $\Delta \mu n>\Omega$. В $[25,26]$ эти неравенства выполняются, поскольку величины сдвига $\Delta^{2} \simeq 8$, ангармонизма $\mu \simeq 10 \mathrm{~cm}^{-1}$ и расстройки ферми-резонанса $\Omega \simeq 5 \mathrm{~cm}^{-1}$.

Таким образом, непертурбативный анализ молекулярного межмодового взаимодействия $\mu$ показывает, что “мягкая" фононная мода $w$ может претерпевать нерегулярное движение вследствие влияния сдвига $\Delta$ электронного терма при электронном переходе. Хаотизация молекулярных спектров может быть результатом этих процессов при включении в корреляционную функцию заданного поля $\psi, \psi^{*}$, как это было сделано 
в гамильтониане $H$ в формуле (37). В этом случае в правой части уравнения (40) появятся внешние силы и хаотизация в системе наступает в соответствии с общей теорией нерегулярной динамики [33].

Роль электрон-фононного взаимодействия в молекуле зависит от его величины, которая определяется величиной сдвига $\Delta$. Для малых сдвигов колебаний связи движение ядер почти гармоническое. Оно дает электронный отклик типа Раби, и электрон сильно взаимодействует с внешним полем. В двухатомных молекулах $\Delta$ мало $(\Delta<1)$ и теория возмущений по $\Delta$ в (38) приводит к обычной теории Ландау-Зинера.

В многоатомной молекуле ситуация такова. При большом сдвиге (деформационное колебание) электронный переход порождает множество фононов, причем ангармоническое взаимодействие $\mu$ между ними сушественно искажает вибрационную структуру спектра. Большая величина $\Delta$ связана с малостью частоты деформационного колебания $\omega$. Если расстройка ферми-резонанса $\Omega$ мала по сравнению с произведением $\mu \Delta$, то электронньй переход вызывает хаос в фононной системе, который приводит к ситуации разупорядочения колебаний при электрон-фононном переходе в молекуле в слабом внешнем поле.

\section{6. ЗАКЛЮЧЕНИЕ}

В данной работе развито непертурбативное низкотемпературное описание квантовых коллективных эффектов, основанное на приближенном анализе двухмасштабной во времени динамики в интегралах по траекториям. Краткий обзор материала представлен в работе [34]. Некоторые комментарии и сравнение с известными подходами в квазиклассике даны ниже.

Разложение Вигнера-Кирквуда [35, 36], представление Вейля [37] и приближение когерентных состояний [38] по большей части формулировались для описания высокотемпературных и высоковозбужденных состояний квантовых систем. Метод самосогласованного эффективного потенциала (в духе работ Фейнмана $[1,39]$ о распределении квазивероятности) был развит для статистической суммы в виде интеграла по траекториям в работе [40]. Основная идея этого метода состоит в интегрировании по квантовым флуктуациям системы при конечной температуре, в то время как классическое поведение тех же степеней свободы исследуется отдельно. Таким способом были рассмотрены модели разреженного газа, ферромагнитной цепочки, синус-Гордон, $\phi^{4}$-поля $[41,42]$ и диссипативных квантовых систем [43].

В отличие от исследований [40-43], в разделах 3 и 5 данной работы рассмотрены сложные системы, в которых "медленные" и "быстрые" степни свободы отнесены к различным типам частиц. Усреднение по “быстрым" степеням свободы определяет масштаб времени для "медленных" частищ, что приводит к низкотемпературному приближению (5). Это приближение выражено неравенствами (23) и (36) в разделах 3 и 5 , трактуюших решетчатые и нуль-мерные задачи.

Разделение "медленных" и “быстрых" степеней свободы в интеграле по траекториям в переменных $\omega, k$ для амплитуды бозона было применено Поповым $[7,44]$ в теории трех- 
мерной сверхтекучести. Была развита теория возмушений для двухточечной равновесной функции Грина и получен функционал энергии для теории сверхтекучести Ландау. Аналогичная идея о "медленных" и "быстрых" переменных использована в разделе 4 данной работы. В отличие от работ $[7,44]$ для статистической суммы "медленной подсистемы" в разделе 4 теория возмушений не использовалась, так что вариационное уравнение для конденсата (26) есть непертурбативное обобщение аналогичного уравнения работ $[7,44]$. Низкотемпературное условие (25) представляет собой ограничение как для $k$ (по образцу работ $[7,44]$ ), так и для $T$ одновременно в соответствии с обшим условием (5). Заметим, что в разделе 4 некоторое количество конденсата присутствует и в разупорядоченной фазе, но оно убьвает до нуля при увеличении температуры. Таким образом, "параметр порядка" $\rho$ принимает участие в формировании высокотемпературного состояния подобно теории работы [17] (в отличие от более ранних рассмотрений бозе-конденсации).

Низкотемпературная квазиклассика для спиновых систем впервые была применена для периодической модели Андерсона в статье [45]. Значение спиновой $(s-d)$-электронной структуры для объяснения эффекта Кондо было отмечено в работе [46]. В разделе 3 дано изложение теории спин-зарядовых переменных в рамках $S U(2 \mid 1)$-когерентного представления хаббардовского $(s)$ электрона. Так, температурная зависимость энергии сдвига $\delta E$ обусловлена полным эффективным взаимодействием между $s$ - и $d$-электронами. Развитие этой техники интегрирования по траекториям в теории димеров можно найти в статье [47]. Применение интегралов по траекториям в когерентном представлении для хаббардовских переменных в теории электронных корреляций обнаруживает преимущество (см. $(t-J)$-модель [48]) по сравнению с традиционной "slave"-бозонной [49] и "slave"- фермионной [50] техникой, приводяшей к проблеме "констрейна" учета связи в пространстве переменных.

Обобщение теории бозе-конденсации Боголюбова [6] для неоднородной среды в координатном представлении можно найти в книге [51]. Современные варианты этого подхода известны под названием ХФБ (Хартри-Фок-Боголюбов) [52] или ХФБ-Попова [53] методов. Модификация этих идей для бозе-конденсации в атомных ловушках на языке связанных нелинейных уравнений Шредингера для конденсата и надконденсата была предложена в работах $[54,55]$. Особое внимание уделялось рассеянию бозонов для сил притяжения и отталкивания. Аналогичные проблемы рассматривались в статье [56].

Обобщение (30), (31) модели Боголюбова (раздел 4) основано на собственно-энергетическом представлении бозонов в неоднородной среде. Это представление позволяет выделить вклад нарушения трансляционной симметрии в наблюдаемые величины. Система вариационных уравнений для плотностей конденсата и надконденсата, приводяшая к эффективному действию для конденсата, служит основным средством рассмотрения сверхтекучести в пористых стеклах.

Что касается больших молекул, экспериментальное размытие оптического спектра длинноволновыми фононами было обнаружено в работе [25] и подтверждено позже [26]. В отличие от известного [57] инфракрасного “теплового взрыва"в молекулах, этот эф- 
фект обнаруживается при электрон-фононных оптических переходах в видимой области спектра. Сравнение этих двух явлений можно найти в статье [30].

Для решения всех рассмотренных в настояшей работе задач были использованы интегралы по траекториям в когерентном представлении. Это представление вводит соответствуюшее данной задаче квазиклассическое фазовое пространство траекторий и дает возможность использовать функциональные методы вычислений.

Квазиклассика для интегралов по траекториям в когерентном представлении $U^{l}(G)$-групп ( $l$ - индекс представления) применялась с параметром $l$ для спина $S U(2)$-модели [58] и в обшем случае в работе [59].

Благодарности. Автор считает своим долгом отметить важное значение его сотрудничества с В. Н. Поповым и Б. С. Непорентом при изучении проблем, затронутых в этой статье. С благодарностью отмечается плодотворная работа с В. Ивановым, Е. Кочетовым, В. Са-Яканитом и М. Журавлевым, а также полезные обсуждения с А. Васильевым, П. Кулишом, Н. Плакидой, В. Приезжевым и Г. Шляпниковым. При подготовке работы автор имел удовольствие пользоваться гостеприимством Г. Глайда в Делаваре и В. Тоньетти во Флоренции.

\section{Список литературы}

[1] R. Feynman, A Hibbs. Quantum Mechanics and Path Integrals. New York: McGrow-Hill Company, 1965.

[2] А.А. Славнов, Л.Д. Фаддеев. Введение в квантовую теорию калибровочных полей. М.: Наука, 1978.

[3] V.N. Popov, V.S. Yarunin. Collective effects in quantum statistics of radiation and matter. Dordrecht: Kluwer, 1988.

[4] E. A. Kochetov, V.S. Yarunin. Physica Scripta. 1995. V. 51. P. 46.

[5] Ю. Изюмов, М. Катцельсон, Ю. Скрябин. Магнетизм электронов. М.: Наука, 1994.

[6] Н. Н. Боголюбов. Изв. АН СССР. Сер. физ. 1947. Т. 11. С. 77.

[7] В. Н. Попов. Континуальные интегралы в квантовой теории поля и статистической физике. М.: Атомиздат, 1976.

[8] E. A. Kochetov. Phys. Lett. A. 1996. V. 217. P. 65.

[9] E. A. Kochetov, V.S. Yarunin, M. Ye. Zhuravlev. SU $(2 \mid 1)$ path integral for heavy electrons in Anderson lattice. In: Proc. of 5-th Intern. Path Integral Conference. Eds. M. A. Smondyrev, V.S. Yarunin. Dubna: JINR, 1996.

[10] E. Kochetov, V. Yarunin, M. Zhuravlev. Physica C. 1998. V. 296. P. 298.

[11] V. Ivanov, R. Pucci, V. Yarunin, M. Zhuravlev. Int. J. Mod. Phys. B. 1997. V. 11. P. 1023.

[12] В. Н. Попов, Л. Д. Фаддеев. ЖЖЭТ. 1964. Т. 47. С. 1315.

[13] В. С. Ярунин. ТМФ. 1993. Т. 96. С. 37.

[14] V.S. Yarunin, L. A. Siurakshina. Physica A. 1995. V. 215. P. 261.

[15] В. С. Ярунин. ТМФ. 1996. Т. 109. С. 295.

[16] N. Blagoveshchenskii, I. Bogoyavlenskii, L. Karnatsevich, Zh. Kozlov, V. Kolobrodov, V. Priezzhev, A. Puchov, A. Skomorokhov, V. Yarunin. Phys. Rev. B. 1994. V. 50. P. 16550.

[17] H. Glyde. Excitations in liquid and solid helium. Oxford: Clarendon Press, 1994.

[18] В. С. Ярунин. Физика низких температур. 1998. Т. 24. С. 130.

[19] V.S. Yarunin. Physica A. 1996. V. 232. P. 436.

[20] Б. Есельсон, Б. Григорьев, Б. Иванцов, Е. Рудавский. Жидкий и твердый гелий. М.: Стандартиздат, 1978. 
[21] V. Sa-Yakanit, V. Yarunin, P. Nisamaneephong. Phys. Lett. A. 1998. V. 237. P. 152.

[22] G. Wong, P. Crovell, H. Cho, J. Reppy. Phys. Rev. Lett. 1995. V. 65. P. 2410.

[23] V. Yarunin. Bose-Einstein condensation of trapped atoms in a gravity field. In: Proc. of NASA/JPL 1998 Workshop on microgravity. USA, Oxnard (accepted).

[24] V. Bagnato, D. Pritchard, D. Kleppner. Phys. Rev. A. 1987. V. 35. P. 4354.

[25] Б. С. Непорент. ЖЭТФ. 1951. Т. 21. С. 172.

[26] Б. С. Непорент, С. В. Куля, А. Г. Спиро. Письма в ЖЭТФ. 1988. Т. 48. С. 367.

[27] Б. С. Непорент, С. А. Федотов, В. С. Ярунин. ЖЭТФ. 1991. Т. 99. С. 447.

[28] Б. Непорент, В. Ярунин. Опт. и спектр. 1990. Т. 69. С. 339.

[29] В. Попов, В. Ярунин. Когерентные коллективные эффекты в сверхпроводимости и нелинейной оптике. Санкт-Петербург: Изд-во Санкт-Петербургского университета, 1994.

[30] V. Yarunin. Physica A. 1993. V. 197. P. 260.

[31] Л. В. Келдыщ. ЖЭТФ. 1964. Т. 47. С. 1515.

[32] J. Schwinger. J. Math. Phys. 1961. V. 2. P. 407.

[33] А. Лихтенберг, М. Либерман. Регулярная и стохастическая динамика. М.: Мир, 1984.

[34] V. Yarunin. Adiabatics and quasiclassics in path integrals for quantum macroscopic phenomena. In: Proc. of 6-th Intern. Path Integral Conference. Florence, Italy (accepted).

[35] M. Hillery, R. O'Connel, M. Scully, E. Wigner. Phys. Rep. 1984. V. 106. P. 122.

[36] E. Wigner. Phys. Rev. 1932. V. 40. P. 749.

[37] Г. Вейль. Теория групп и квантовая механика. М.: Наука, 1986.

[38] J. R. Klauder. Coherent states. Singapore: World Scientific, 1985.

[39] P. Фейнман. Статистическая механика. М.: Мир, 1975.

[40] A. Cuccoli, V. Tognetti, R. Vaia. Phys. Rev. A. 1992. V. 45. P. 8418.

[41] A. Cuccoli, R. Giachetti, V. Tognetti, R. Vaia, P. Verrucchi. J. Phys.: Conds. Matter. 1995. V. 7. P. 7891.

[42] A. Cuccoli, R. Livi, M. Spissi, V. Tognetti, R. Vaia. Int. J. Mod. Phys. B. 1994. V. 8. P. 2391.

[43] A. Cuccoli, A. Rossi, V. Tognetti. Phys. Rev. E. 1997. V. 55. P. R4849.

[44] V. N. Popov. Functional integrals and collective excitations. Cambridge: Cambridge University Press, 1987.

[45] V.S. Yarunin, M. Ye. Zhuravlev. Effective hopping for localized electrons in periodic Anderson model. In: Thes. Intern. Seminar "Strongly correlated systems". Ed. V.S. Udovenko. Dubna: JINR, 1994.

[46] А. Ф. Барабанов, А. М. Цвелик. ФТТ. 1979. Т. 21. С. 3214.

[47] R. Pucci, V. Yarunin, M. Zhuravlev. J. Phys. A. 1998. V. 31. P. 3185.

[48] E. Kochetov, V. Yarunin. Phys. Rev. B. 1997. V. 56. P. 2703.

[49] P. Coleman. Phys. Rev. B. 1984. V. 29. P. 3035.

[50] C. Kane, P. Lee. Phys. Rev. B. 1989. P. 6880.

[51] P. R. de Gennes. Superconductivity of metals and alloys. New York: Benjamin, 1966.

[52] A. Minguzzi, M. Chiofalo, M. Tosi. Phys. Lett. A. 1997. V. 236. P. 237.

[53] R. Dodd, M. Edwards, C. Clark, K. Burnett. Collective excitations of Bose-Einstein condensed gases at finite temperatures. cond-mat/9708139, 19 Aug. 1997.

[54] Yu. Kagan, G. Shlyapnikov, J. Warlaven. Phys. Rev. Lett. 1996. V. 76. P. 2670.

[55] Yu. Kagan, E. Surkov, G. Shlyapnikov. Phys. Rev. A. 1996. V. 54. P. R1753.

[56] C. Law, H. Pu, N. Bigelow, J. Eberly. Phys. Rev. Lett. 1997. V. 79. P. 3105.

[57] М. В. Кузьмин, В. С. Летохов, А. А. Стучебрюхов. ЖЭТФ. 1986. Т. 90. С. 458.

[58] E. Kochetov. J. Math. Phys. 1995. V. 36. P. 4667.

[59] E. Kochetov. J. Phys. A. 1998. V. 31. P. 4473. 